\title{
Efecto de la transformación geométrica del fruto de melón (Cucumis melo L) sobre su calidad bajo cultivo sin suelo
}

\author{
Effect of the geometric transformation of the fruit of the melon \\ (Cucumis melo L) on its quality under soilless cultivation
}

\author{
Andrea Retamal C., Leonardo Vásquez Ch., Pilar Mazuela A. ${ }^{1}$
}

\section{RESUMEN}

En los últimos años el consumo de productos Gourmet ha ido creciendo a una tasa de $10 \%$ anual, siendo cada vez más cotizados por los consumidores. Esta tendencia se ve marcada en mercados como el europeo y norteamericano. Bajo esta situación el desarrollo de productos con valor agregado tiene una importancia mayor en zonas desérticas con escasa disponibilidad de tierras y agua en los cuales se busca aumentar su rentabilidad. El objetivo de este estudio fue evaluar el efecto de la trasformación geométrica en frutos de melón (Cucumis melo L.) sobre los parámetros de calidad en dos cultivares en dos experimentos: cv. Amarillo oro y cv. DRG 2099 cv. Se cultivó en un sistema de cultivo sin suelo, utilizando perlita como sustrato. Los tratamientos fueron, T0: frutos sin molde y T1: frutos con molde. Los parámetros de calidad evaluados fueron Sólidos Solubles Totales, firmeza de frutos, acidez titulable y pH. No se observaron diferencias significativas en la calidad de frutos entre tratamientos. En cuanto al manejo agronómico se logró desarrollar una técnica basada en los periodos determinantes en la formación de los frutos, concluyendo que sí es posible transformar la geometría de frutos de melón (Cucumis melo L.) sin afectar su calidad.

Palabras clave: cultivo sin suelo, invierno, melones cuadrados, perlita.

\begin{abstract}
In recent years the consumption of gourmet products has been increasing by nearly $10 \%$ per year; these products are increasingly appreciated by consumers. This tendency is especially notable in the European and North American markets. In these circumstances, the development of products with added value is more important in areas with limited availability of soils and water such as arid zones, in order to improve the profits. The objective of this study was to obtain square fruits of melon (Cucumis melo $L$.), evaluating the effects produced by transforming its geometry on quality parameters in the varieties Amarillo Oro and DRG 2099. The crops were grown in soilless culture, using Perlite as substrate. The treatments were T0, fruits without mold and T1, fruits with mold. The quality parameters evaluated were total soluble salts, fruit firmness, titratable acidity and $\mathrm{pH}$. The results showed no significant differences in fruit quality between treatments. In terms of agronomic management, we developed a technique based on determinant periods in fruit formation, concluding that it is possible to transform the geometry of melon fruits (Cucumis melo L.) without affecting their quality.
\end{abstract}

Key words: soilless culture, winter, melon squares, perlite.

\section{Introducción}

Arica presenta condiciones excepcionales en el cultivo de hortalizas, bien sea como primor, para el consumo en fresco en contraestación para la zona central; como proveedor de productos Gourmet, donde se valora la exclusividad; como productor de almácigos para proveer de plantas a los productores de los valles costeros y del sur de Perú, así como para la producción de semillas (Mazuela et al., 2009). El mayor capital hortícola que tiene la Región de Arica y Parinacota es el clima que permite la producción de hortalizas durante todo el año. Predominan las condiciones de clima de desierto costero con nubosidad abundante, ausencia de heladas, vientos moderados, alta humedad relativa y radiación solar directa durante todo el año. La temperatura media anual es de $18{ }^{\circ} \mathrm{C}$, máximas medias anuales de $23,6^{\circ} \mathrm{C}$ y mínimas de $13,8^{\circ} \mathrm{C}$ (Torres y Acevedo, 2008). La región abastece a una importante parte del país con sus productos fuera de temporada, como es el caso del tomate (Saavedra y Tapia, 2009).

Ante la posibilidad de diversificar la agricultura existen excelentes perspectivas para productos como

1 Universidad de Tarapacá, Arica, Chile.pmazuela@uta.cl 
el melón, entre otras hortalizas, para ser incorporado en mayores superficies de cultivo (Mazuela, 1999). La producción de melón en la comuna de Arica entre los años 1999 y 2007 se ha incrementado, pasando de 6,78 a 12,82 ha (INE, 2007). Considerando la importancia de acceder a nuevos mercados con productos de valor agregado, una alternativa es cambiar la geometría del fruto para facilitar así el almacenaje y optimizar su traslado. En la actualidad, la población mundial ha aumentado su grado de exigencia respecto a los productos alimenticios que consumen, lo que se debe principalmente al acceso a información y también al aumento de la oferta de productos diferenciados. Por lo tanto, se ha observado que existe una tendencia creciente a consumir productos gourmet en los diferentes mercados (PRO CHILE, 2009).

\section{Materiales y Métodos}

Para el desarrollo de la investigación se ocuparon las instalaciones de la Facultad de Ciencias Agronómicas, ubicadas en el km 12 del Valle de Azapa (18³1'15"S 70¹0'42”'O), a 250 m.s.n.m. en la XV Región de Arica y Parinacota, en una superficie de $220 \mathrm{~m}^{2}$ en un invernadero de malla antiáfido. Los tratamientos se realizaron en sistemas de cultivo sin suelo para optimizar el uso del recurso hídrico y llevar un mayor control sobre los parámetros de fertirriego. El material vegetal utilizado fue melón Cucumis melo L. var. Saccharinus, cv. amarillo oro y var. Cantalupensis, cv. DRG 2099. El cultivo se realizó entre junio y septiembre del 2010. Se trabajó con 80 macetas de $5 \mathrm{~L}$ cada una, dispuestas en 8 líneas. El marco de plantación fue de $0,8 \mathrm{~m}$ sobre la hilera y $1,5 \mathrm{~m}$ entre hilera. Durante el desarrollo vegetativo del cultivo se incorporó el uso de una manta térmica para aumentar la temperatura y mejorar las condiciones ambientales en los melones de invierno. Para asegurar la polinización y determinar el momento en que ésta acontece en invierno, se recurrió a la del tipo manual, a los 50 días después del trasplante (DDT). La conductividad eléctrica (CE) media del agua de riego fue de $0,8 \mathrm{dS} \mathrm{m}^{-1} \mathrm{y}$ $\mathrm{pH} 7,1$. La CE de la disolución nutritiva utilizada fue de $2,7 \mathrm{dS} \mathrm{m}^{-1}$ y pH 5,7. La composición media de los nutrientes aportados fue: $10 \mathrm{de} \mathrm{NO}_{3}^{-} ; 1,5$ de $\mathrm{H}_{2} \mathrm{PO}_{4}^{--} ; 3,5$ de $\mathrm{SO}_{4}{ }^{2-} ; 6$ de $\mathrm{K}^{+} ; 8,5$ de $\mathrm{Ca}^{2+}$ y 3,6 de $\mathrm{Mg}^{2+}$, expresados en me $\mathrm{L}^{-1}$. La recolección se realizó en dos ocasiones, a los 95 y a los 103 días después del trasplante (DDT). Para detectar el grado de madurez adecuado de cosecha de los frutos se utilizó como índice de madurez, el color el cual dependió de los cultivares utilizados.

\section{Parámetros de calidad}

Se evaluaron firmeza $(\mathrm{kg})$ de fruto y sólidos solubles totales ( ${ }^{\circ}$ Brix) de frutos, acidez titulable y pH. La firmeza de fruto a la presión se determinó con un penetrómetro marca Effegi modelo FT 011 (0-11 lb), utilizando un émbolo de $8 \mathrm{~mm}$ de diámetro. La concentración de sólidos solubles se midió con un refractómetro portátil termocompensado marca Arquimed modelo 2003319. El pH se determinó usando un $\mathrm{pH}$-metro portátil con electrodo de vidrio. En cuanto a la acidez titulable, se determinó por titulación con $\mathrm{NaOH} 0,1 \mathrm{~N}$. Los resultados fueron expresados como porcentaje (\%) de ácido cítrico según las Normas Covenin 1984 (García, 2009).

El grado de formación de frutos se determinó midiendo el volumen $(\mathrm{mL})$ de los frutos correspondientes al tratamiento T1, en ambos experimentos. Se depositó cada fruto en un recipiente previamente graduado, determinándolo por desplazamiento de volumen y se relacionó con él de almacenaje de los moldes. Los resultados fueron expresados en porcentaje (\%). Este parámetro se creó con la finalidad de caracterizar el grado que los frutos adoptaron la forma del molde, para lo cual se diseñó una escala (Figura 2) que se divide en cuatro categorías de acuerdo al grado de formación (Tabla 1). Los frutos que se emplearon para el análisis de calidad corresponden a las primeras dos categorías.

El diseño experimental para cada experimento fue de bloques completamente al azar (Little y Hills, 1976; Petersen, 1994) con dos tratamientos y cinco repeticiones. Para la separación de medias se usó la probabilidad asociada a la t de Student. Para los cálculos se utilizó el programa Microsoft Excel 2000.

Los tratamientos considerados fueron: $\mathrm{T}_{0}$ : fruto sin molde y $\mathrm{T}_{1}$ : fruto con molde.

\section{Resultados y Discusión}

Los resultados de los parámetros de calidad del fruto se indican en la Tabla 2. En el experimento 1 (cv. amarillo oro) no se encontraron diferencias significativas entre tratamientos para sólidos solubles totales (SST), firmeza de fruto y $\mathrm{pH}$. En SST, los valores obtenidos en los tratamientos $\mathrm{T}_{0}$ 
y $\mathrm{T}_{1}$ fueron: 13,6 y $12,9^{\circ}$ Brix, respectivamente, similares a los indicados por Castoldi et al. (2008) y considerados de excelente calidad por otros autores (Odet, 1992; Gorgatti Neto et al., 1994; Lee-Jones et al., 1997), para un cultivo de melón. Los valores de $\mathrm{pH}$ concuerdan con los indicados por Rizzo y Braz (2004) y García et al. (2009). En acidez titulable (AT), se encontraron diferencias altamente significativas entre tratamientos, siendo el tratamiento $\mathrm{T}_{1}(0,217 \%$ ácido cítrico) el que obtuvo el mayor contenido de AT en comparación $\mathrm{a} \mathrm{T}_{0}(0,137 \%$ ácido cítrico). Estos resultados están dentro de los valores encontrados por Mendlinger (1992) que van desde los 0,051 a 0,35\% de ácido.
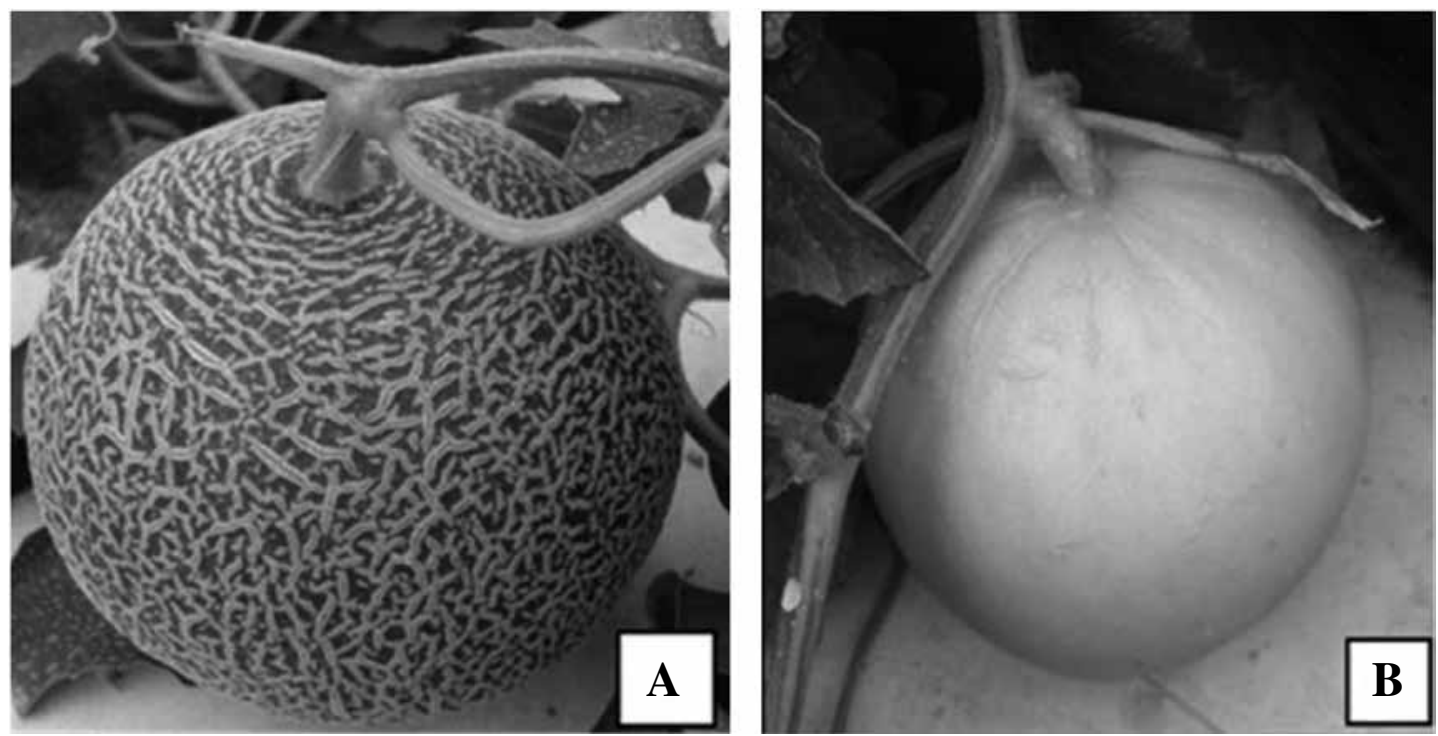

Figura 1. Material vegetal utilizado en invierno. Cucumis melo L.: (A) var. Cantalupensis, cv. DRG 2099; (B) var. Saccharinus, cv. Amarillo oro.
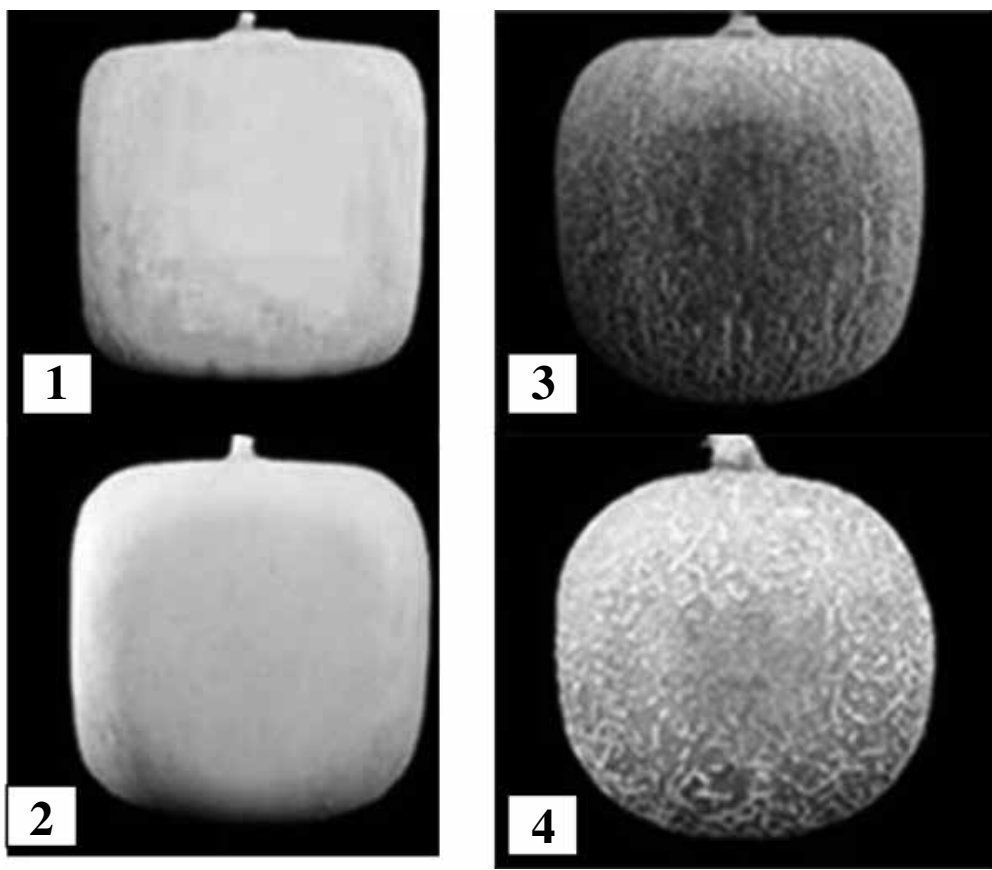

Figura 2. Frutos de Cucumis melo L. según la escala de clasificación para melones cuadrados. 
Tabla 1. Escala de clasificación para melones cuadrados según el grado de formación.

\begin{tabular}{ccl}
\hline Categoría & $\begin{array}{c}\text { Grado de formación } \\
(\%)\end{array}$ & \multicolumn{1}{c}{ Descripción } \\
\hline I & $>85$ & $\begin{array}{l}\text { Frutos comprendidos en un rango mayor a 85\%, poseen una forma con bordes muy } \\
\text { definidos y cumplen con todas las características deseadas en el ensayo. }\end{array}$ \\
\hline II & $85-80$ & $\begin{array}{l}\text { Comprende el rango entre 85 y 80\%. Son frutos con forma definida y bordes un } \\
\text { tanto circulares. Esta escala junto a la anterior cumplen con las características } \\
\text { buscadas en el ensayo. }\end{array}$ \\
\hline III & $80-75$ & $\begin{array}{l}\text { Esta escala no posee las características deseadas. Comprende el rango entre } 80 \text { y } \\
75 \% \text {. Son frutos con forma poco definida. En esta escala están presentes aquellos } \\
\text { con bordes irregulares (frutos mal formados). }\end{array}$ \\
\hline IV & $<75$ & $\begin{array}{l}\text { Frutos comprendidos en un rango inferior a 75\%. Comprende aquellos que alcanzaron } \\
\text { solo a marcarse las caras del molde, con una apariencia más circular que cuadrada, } \\
\text { hasta aquellos que no lograron cambiar de forma. El grado de formación es casi nulo. }\end{array}$ \\
\hline
\end{tabular}

Fuente: Elaboración propia.

Tabla 2. Variables de calidad para un cultivo de melón cv. amarillo oro (Experimento 1) y cv DRG 2099

(Experimento 2), según tratamiento: sin transformación geométrica (T0), con transformación geométrica (T1), en cultivo sin suelo.

\begin{tabular}{|c|c|c|c|c|c|c|c|c|}
\hline & \multicolumn{4}{|c|}{ cv. Amarillo Oro } & \multicolumn{4}{|c|}{ DRG 2099} \\
\hline & SST & $\mathrm{FF}$ & $\mathrm{pH}$ & AT & SST & $\mathrm{FF}$ & $\mathrm{pH}$ & AT \\
\hline T0 & 13,6 & 5,85 & 6,3 & 0,134 & 12,6 & 8,4 & 6,6 & 0,220 \\
\hline $\mathrm{T} 1$ & 12,9 & 6,35 & 5,9 & 0,217 & 13,7 & 6,6 & 6,6 & 0,286 \\
\hline $\mathrm{p}$ & $\mathrm{ns}$ & $\mathrm{ns}$ & $\mathrm{ns}$ & $* *$ & $* *$ & $* *$ & $\mathrm{~ns}$ & $\mathrm{~ns}$ \\
\hline
\end{tabular}

$*, * *, * * *, n s$, son $\mathrm{p} \leq 0,05, \mathrm{p} \leq 0,01, \mathrm{p} \leq 0,001$ y no significativo.

SST: Sólidos Solubles Totales ( ${ }^{\circ}$ Brix); FF: Firmeza de Fruto (kg); pH; AT: Acidez Titulable: Ac (\% ácido cítrico).

En el Experimento 2 (cv. DRG 2099), los resultados obtenidos muestran diferencias altamente significativas para el parámetro de SST, siendo el $\mathrm{T}_{1}$ (frutos con molde) el que presentó un mayor contenido de azúcares con $13,7^{\circ}$ Brix en comparación a los $12,6^{\circ}$ Brix que presentó $\mathrm{T}_{0}$. Estos resultados coinciden con los obtenidos por Lainez y Krarup (2008) y Castoldi et al. (2008). Estos resultados sugieren que el esfuerzo en la transformación geométrica puede causar un estrés en el fruto provocando un aumento de sólidos solubles, debido a diferencias en los factores microambientales por tratamiento. Weller y Buitelaar (1988) mencionan a la temperatura como un factor ambiental que acelera las reacciones metabólicas, la cual influye sobre la acumulación de azúcares, aumentando así el contenido de sólidos solubles del $\mathrm{T}_{1}$. En cuanto a firmeza de frutos (FF), también existen diferencias altamente significativas. En $\mathrm{T}_{0}$ presentó una mayor firmeza de la pulpa con $8,4 \mathrm{~kg}$ en comparación al $\mathrm{T}_{1}$, el cual presentó una firmeza de 6,6 kg. Estos valores son mayores a los obtenidos por Mazuela (2009) y pueden atribuirse a la menor temperatura ambiental durante la maduración del fruto considerando que Gil (2004) menciona a la temperatura como uno de los factores que influyen en el ablandamiento de la fruta. La FF es de gran importancia pues incide directamente en la resistencia al transporte y en la aceptación por parte del consumidor, que, en general, prefiere pulpas firmes antes que blandas y acuosas (Ribas et al., 2002).

\section{Conclusión}

Según los resultados obtenidos en los experimentos para determinar el efecto que produce 
transformar la geometría de frutos de melón en la calidad se concluye que esto depende principalmente de la variedad a utilizar. En cv DRG 2099 el comportamiento fisiológico durante su crecimiento afectó la calidad asociada a los frutos sometidos a transformación geométrica en menor contenido de sólidos solubles totales y menor firmeza, lo que sugiere que al hacer crecer los frutos en un espacio confinado y con poca ventilación se produce una aceleración en el metabolismo, induciendo su precocidad. Con respecto a la obtención de melones cuadrados los resultados sugieren que depende de la variedad utilizada, debido a su comportamiento fisiológico durante su crecimiento, ya que variedades con un alto contenido de intercambio gaseoso como el cultivar DRG 2099 necesitan un sistema de ventilación en el molde para evitar su pudrición. Es necesario conocer el tamaño de los frutos para estimar el tamaño del molde a utilizar.

En general, se concluye que es posible hacer melones cuadrados sin que se afecte la calidad del fruto y el manejo dependerá de la variedad de melón a utilizar.

\section{Agradecimientos}

Este trabajo ha sido financiado por el Proyecto de Investigación Estudiantil UTA 9765-10.

\section{Bibliografía}

Castoldi, R.; De Oliverira, H.; Forlan, P.; Trevizan, L.

2008. Qualidade de frutos de cinco híbridos de melão rendilhado em função do número de frutos por planta. Rev. Bras. Frutic., Jaboticabal - SP, V. 30 (2), pp. 455-458.

Gil, G.

2004. Madurez de la fruta y manejo poscosecha. Agronomía y Forestal. Universidad Católica. 432: pp. 224-431.

Gorgatti Neto, A.; Gayet, J.P.; Bleinpoth, E.W.; Matallo, M.; Instituto Nacional de Estadísticas

2008. VII Censo Agropecuario y Forestal 2006-2007. Resultados preliminares. INE Ediciones, Santiago de Chile, 444 p.

Lainez, D.; Krarup, C.

2008. Caracterización en pre y poscosecha de dos cultivares de melón reticulado del tipo Oriental (Cucumis melo Grupo Cantalupensis). Rev. Ciencia e Investigación Agraria. 35 (1): 59-66.

Lee-Jong, N.A.M.; Lee-Jeong, T.A.E.; Jang-Sukwoo; Kim-

Wonbae; O.M., Y.H. Y Pak, H.Y.

1997. Growth and yield of muskmelon (Cucumis melo L) by the different mulches under rainshelter in Alpine area. Horticulture-Science. 39 (2): 49-55.

Little, T.; Hills, F. 1976. Métodos estadísticos para la investigación en la agricultura. Ed. Trillas, México. 270 p.

Mazuela, P.

1999. Desarrollo agrícola en Arica: Innovación y cambio de mentalidad. Temas Regionales V. 6. pp. 156-166.

Mazuela, $\mathrm{P}$.

2009. Effect of oxygen supply on water uptake in a melon crop under soilless culture. Interciencia. Asociación Interciencia. Caracas, Venezuela. Vol. 35 (10), pp. 769-771.
Mendlinger, S.

1992. Effect of increasing plant density and salinity on yield and fruit quality in muskmelon. Scientia Horticulturae. V57, Issues 1-2, pp. 41-49.

Odet, J.

1992. Le Melon. París: CTIFL de 1992, pp. 295.

Petersen, R.G.

1994. Agricultural Field Experiments. Marcel Dekker Inc., New York, pp. 409.

PRO CHILE.

2009. Estudio de mercado Industria Gourmet en Chile. Santiago-Chile. Pp. 122.

Ribas, F.; Cabello, M.; Moreno, M.; Moreno, A.; López-Bellido, L. 2002. Respuesta fisiológica de un cultivo de melón (Cucumis melo L) a distintas dosis de riego. Invest. Agr. Prod. Prot. Veg. Vol. 15 (3).

Rizzo, A.A.; Braz, L.T.

2004. Desempenho de linahagens de melão rendilhado em casa de vegetação. Hortic. Bras. 22 (4): 784-788.

Saavedra, A.; Tapia, L.

2009. Evaluación de las estrategias de producción de tomate (Lycopersicum esculentum Mill) fuera de estación para la agricultura de la provincia de Arica, Chile, entre los años 1995-2005. Idesia 27 (2): 91-96.

Torres, A.; Acevedo, E.

2008. El problema de salinidad en los recursos suelo y agua que afectan el riego en los valles de Lluta y Azapa en el norte de Chile. Idesia 26 (3): 31-44.

Weller, G.W.H.; Buitelaar, K.

1988. Factores que afectan el contenido de sólidos solubles de melón (Cucumis melo L.). Países Bajos Diario de Ciencias Agrícolas, Vol. 36, pp. 239-246. 
